East African Medical Journal Vol. 81 No. 3 March 2004

MISSED OPPORTUNITIES AND INAPPROPRIATELY GIVEN VACCINES REDUCE IMMUNISATION COVERAGE IN FACILITIES

THAT SERVE SLUM AREAS OF NAIROBI

P. K. Borus DrPH., Senior Research Officer, Centre for Virus Research, Kenya Medical Research Institute, P.O Box 54840, Nairobi, Kenya

\title{
MISSED OPPORTUNITIES AND INAPPROPRIATELY GIVEN VACCINES REDUCE IMMUNISATION COVERAGE IN FACILITIES THAT SERVE SLUM AREAS OF NAIROBI
}

\author{
P. K. BORUS
}

\begin{abstract}
Objectives: To quantify missed opportunities for immunisation, document reasons for their occurrence and evaluate the extent of inappropriately given vaccine doses.

Design: A cross sectional study of children under two years of age attending health facilities.

Setting: Six health facilities predominantly serving the slums of Nairobi.

Methodology: Information on vaccination was extracted from child immunisation cards as well as from mothers or guardians of children.

Results: Effective immunisation coverage for Bacille-Callmette Guerin (BCG) was $91 \%$. Coverage for the birth dose, first, second, and third doses of oral polio vaccine (OPV0, OPVI, OPV2, and OPV3) was $44 \%, 83 \%, 79 \%$ and $75 \%$ respectively. Effective coverage for first, second and third doses of diphtheria-pertusis-tetanus (DPTI, DPT2 and DPT3) vaccine was $88 \%, 87 \%$ and $85 \%$ respectively. Measles coverage was $80 \%$.

Immunisation coverage for all antigens except OPV0 and OPV3 would have been increased to over $90 \%$ had missed immunisation opportunities and inappropriately administered vaccination been avoided. There would have been an $11 \%$ increase in OPV3 coverage to $86 \%$. Increases in coverage for OPVI and OPV2 would have been $16 \%$ and $18 \%$ respectively. Coverage would have increased by $10 \%$ for diphtheria pertusistetanus (DPT) doses DPTI and DPT2, and $7 \%$ for DPT3. Measles immunisation coverage would have increased by $19 \%$ had missed immunisation opportunities and inappropriately administered vaccinations been avoided. The overall missed opportunities rate was $3 \%$. The proportions of missed opportunities were higher for the OPV series than DPT series. Conclusion: Missed immunisation opportunities among clinic attendees in Nairobi occur and routine supervision should be strengthened in these health facilities in order to minimise such missed opportunities and inappropriately administered vaccines.
\end{abstract}

\section{INTRODUCTION}

The World Health Organisation's Expanded Programme on Immunization (EPI) recommends that children be vaccinated at every contact with a health facility(1). Failure to vaccinate a child who attends immunisation or curative clinics with vaccine(s), for which he/she is eligible in the absence of any known contra-indication, constitutes a missed immunisation opportunity.

Missed immunisation opportunities arise because of system factors such as non availability of vaccines, failure by facilities to immunise on all days of the week, or negative parental beliefs that prevent them from allowing their children to be vaccinated. When there is a true or absolute contraindication to vaccination, failure to immunise does not, by definition, constitute a missed immunisation opportunity. True contraindications of vaccination include complications arising from use of vaccines. Although the rate of occurrence of these complications is very rare, convulsions may, for example, follow DPT or measles immunisation(2). DPT vaccination should not be given if a previous dose resulted in severe reactions such as shock, high fever, convulsions, neurological conditions or anaphylactic shock. It is recommended for sick children whose conditions require hospital admission, that immunisation is deferred so that the decision to immunise is made by the admitting hospital. This is to avoid difficulties in subsequent diagnosis should a child's condition worsen after immunisation(3). The EPI recommends that children who had not received relevant doses are eligible for immunisation even if they present in health facilities with low-grade fever, mild respiratory illness, diarrhoea and other minor illnesses that are considered as false contraindications.

The routine immunisation schedule in developing countries aims to avail vaccines before ages when 
children are at risk of disease and includes administration of BCG and OPV at birth. During recommended visits at 6, 10 and 14 weeks, DPT and OPV are given whereas measles is given at nine months(4). The interval between attenuated live vaccine doses in this schedule should be four weeks. The basis for the selection of the primary immunisation schedule has been extensively reviewed(5). Inappropriate doses as referred to in this study are those given either earlier than the recommended time, or for attenuated live antigen doses, when the intervals between doses were less than four weeks.

Determination of the extent and reasons for missed immunisation opportunities gives an estimate of the magnitude of the opportunity that exists for the direct increase of immunisation coverage. On the other hand, inappropriately given doses could be less effective due to viral interference. Thus, these could be discounted in a 'worst-case' scenario when calculating vaccine coverage levels. The proportion of missed immunisation opportunities and inappropriately given doses has been calculated during coverage surveys and through exit interviews of mothers as they leave health facilities with their children(6).

A large amount of literature exists on the extent and reasons for missed opportunities. Most of these come from surveys in developing countries and poor inner cities in developed countries, reflecting on the emphasis attached to improving coverage in underserved populations. In a review of missed opportunities in developing and developed countries, Hutchins and colleagues(7) found the reasons for missed opportunities to be similar in both settings, variations being in their prevalence. This variation is understandable because there is bound to be differences in EPI infrastructure and provider-clientele characteristics in both settings. Failure to immunise for vaccines that are given on a single visit in the routine schedule i.e. OPV and DPT at 6,10 and 14 weeks, was reported as a common reason for missed immunisation opportunities. Other reasons were false contraindications of immunisation as well as improper health worker practices including failure to open a multi-dose vial for a small number of children. Other reasons included weaknesses in logistics that lead to vaccine shortage, poor clinic organisation and inefficient scheduling of vaccination. Parental refusal to let a child be vaccinated was not a common reason. A small study involving 23 children in the first decade of the existence of the Kenya Expanded Programme on Immunisation (KEPI) reported $4 \%$ of children were missed for immunisation when they attended an outpatient $\operatorname{clinic}(8)$.

This study was primarily designed to calculate the rate of and reasons for missed immunisation opportunities. The number of inappropriate doses given by facilities were assessed to determine the increase in immunisation coverage that would be achieved if these were correctly given.

\section{MATERIALS AND METHODS}

The study was a cross-sectional assessment of missed immunisation opportunities in six health facilities in Nairobi. These facilities were four health centres (African Medical and Research Foundation - AMREF, Baba Dogo, Kayole, Mukuru), one hospital (Mbagathi) and one clinic (Pangani). The study was carried out between April and May 2001. The choice of facilities was made purposively to reflect on facilities that serve slum areas of the city.

Children under two years of age attending these health facilities were included in the study and their mothers or adult carers of children were interviewed. The reference population of these children for each facility for use in sample size calculation was determined using figures available from the facilities or from census data. A minimum sample size of 51 children was required for each facility as determined using EPI INFO software. The calculation assumed missed opportunities rate of $16 \%$ (for the primary objective), a precision of $10 \%$ and $95 \%$ confidence level.

The KEMRI ethical committee approved the study and written permission was given by the medical officer/ administrator in-charge of facilities. Informed consent was sought from the mother or guardian of participating children. A questionnaire was used to collect information on documented immunisation as well as reasons for a child's visit, mother's knowledge of and attitudes towards immunisation, as well as information on costs relating to immunisation, and in particular on supplies such as syringes and needles. The timing of vaccination as well as intervals between doses of the same antigen were noted in order to determine inappropriately administered vaccines.

Whenever possible, interview days in the facilities were planned for the same days of the week or were synchronised to take place on days when the same antigens were given across facilities. The days spent on a facility were minimised to avoid influencing clinic practice and routines.

\section{RESULTS}

Four hundred and eighteen children participated in the study. Routine immunisation coverage for the primary EPI series was assessed by extracting information from child immunisation cards and by maternal recall of vaccination. Four hundred and fourteen $(99 \%)$ of respondent mothers claimed a child they came with had an immunisation card. This was confirmed in 409 (98\%) children by physical inspection.

The proportion of children who were validly vaccinated for each antigen was determined. Valid vaccine doses are those administered at or after the recommended age of vaccination i.e. 42 days for $\mathrm{OPVI} /$ DPTI, 70 days for OPV2/DPT2, 98 days for OPV3/ DPT3 and 274 days for measles vaccine and within two weeks of birth for OPV0. Coverage ror DPTI, DPT2 and DPT3 was $88 \%, 87 \%$ and $85 \%$ respectively compared to $83 \%, 79 \%$ and $75 \%$ for OPV1, OPV2 and OPV3. Measles immunisation coverage was $80 \%$. The median ages for DPT1, DPT2 and DPT3 immunisation were 47, 80 and 115 days respectively. The median age of measles immunisation was 290 days. 
Table 1

Vaccine coverage and missed opportunities

\begin{tabular}{|c|c|c|c|c|c|c|c|c|c|c|c|}
\hline \multirow[t]{2}{*}{ Antigen } & \multirow[b]{2}{*}{$\mathrm{N}$} & \multicolumn{3}{|c|}{ Vaccinated at correct age } & \multicolumn{3}{|c|}{ Missed Opportunities } & \multicolumn{2}{|c|}{$\begin{array}{l}\text { Early or } \\
\text { inappropriate } \\
\text { doses }\end{array}$} & \multicolumn{2}{|c|}{$\begin{array}{c}\text { Potential } \\
\text { coverage by avoiding } \\
\text { MIO \& ID }\end{array}$} \\
\hline & & $\mathrm{n}$ & $\%$ & $\begin{array}{l}\text { Median } \\
\text { age }\end{array}$ & $\mathrm{n}$ & $\%$ & $\begin{array}{l}\text { PC- } \\
\text { MIO }\end{array}$ & $\mathrm{n}$ & $\%$ & $\mathrm{n}$ & $\%$ \\
\hline $\mathrm{BCG}$ & 418 & 384 & 91.9 & 17 & 20 & 4.8 & 96.7 & 0 & 0 & 404 & 96.7 \\
\hline OPVO & 392 & 173 & 44.1 & 4 & 3 & 0.7 & 45.7 & 0 & 0 & 179 & 45.7 \\
\hline OPV1 & 340 & 282 & 82.9 & 47 & 15 & 4.4 & 87.3 & 41 & 12 & 338 & 99.4 \\
\hline OPV2 & 289 & 228 & 78.9 & 82 & 16 & 5.5 & 84.4 & 30 & 10 & 274 & 94.8 \\
\hline OPV3 & 247 & 186 & 75.3 & 117 & 14 & 5.7 & 81.0 & 14 & 6 & 214 & 86.6 \\
\hline DPT1 & 340 & 298 & 87.6 & 47 & 2 & 0.6 & 88.2 & 33 & 10 & 333 & 97.9 \\
\hline DPT2 & 289 & 249 & 86.2 & 80 & 2 & 0.7 & 86.9 & 26 & 9 & 277 & 95.8 \\
\hline DPT3 & 247 & 210 & 85.0 & 115 & 1 & 0.4 & 85.4 & 15 & 6 & 226 & 91.5 \\
\hline Measles & 107 & 86 & 80.4 & 290 & 4 & 3.7 & 84.1 & 16 & 15 & 106 & 99.1 \\
\hline
\end{tabular}

$\mathrm{N}=$ Denominator or number of children eligible for immunisation, $\mathrm{n}=$ Numerator, $\mathrm{PC}-\mathrm{MIO}=$ Coverage by avoiding missed opportunities, $\mathrm{MIO}$ and $\mathrm{ID}=$ Missed immunisation opportunities and early/inappropriately administered vaccination

The proportion of children aged 12-23 months old who were fully immunised was assessed. The median age of these children was 455 days ( 15 months). Of 23 children who were aged over one year old, 19 were fully immunised, that is, each had received one dose of BCG, three valid doses of OPV, three valid doses of DPT and one valid dose of measles vaccine.

Missed immunisation opportunities were assessed as a proportion of age-eligible children who were attended to at surveyed health facilities for various reasons over interview days. The overall missed opportunities rate was 3\% (77/2659). Missed immunisation opportunities were more common for oral polio vaccines. The proportion of missed opportunities for OPV1, OPV2 and OPV3 was $4.4 \%, 5.5 \%$ and $5.7 \%$ respectively (Table 1). In contrast, the proportion of missed immunisation opportunities for the DPT series was $0.6 \%, 0.7 \%$ and $0.4 \%$ for DPT1, DPT2 and DPT3 respectively. The proportion of missed opportunities to immunise for measles was $3.7 \%$.

Had all opportunities to immunise been utilised, immunisation coverage for BCG would have increased to $97 \%$. Similarly coverage for OPV0, OPV1, OPV2 and OPV3 would have increased to $46 \%, 87 \%, 84 \%$ and $81 \%$ respectively. For the DPT series, coverage would have increased only marginally. Measles immunisation coverage would have increased to $84 \%$.

Immunisation coverage for all antigens except OPV0 and OPV 3 would have been increased to over 90\% had missed immunisation opportunities and inappropriately given vaccines been avoided. There would have been $16 \%, 18 \%$ and $11 \%$ increase in coverage for OPV1, OPV2 and OPV3; whereas DPT1 and DPT2 coverage would have increased by $10 \%$. DPT3 and measles coverage would have increased by $7 \%$ and $19 \%$ respectively.

Missed immunisation opportunities were also assessed by health facility (Table 2). The proportion of missed immunisation opportunities varied according to facility. Baba Dogo and Kayole had high proportions of missed immunisation opportunities for OPV. AMREF had only missed opportunities for BCG and not other antigens. Mbagathi, Mukuru and Pangani never had missed opportunities for the majority of antigens. Mukuru had the highest proportion (11\%) of missed opportunities for BCG vaccination. Missed opportunities for OPV1 and OPV2 occurred only in Baba Dogo and Kayole where the proportions were $7.4 \%$ and $18 \%$, and $9.6 \%$ and $23.9 \%$ respectively. In addition, the proportion of missed opportunities for OPV3 in Baba Dogo, Kayole and Mukuru were 19\%, 10\% and 3\% respectively. 
Table 2

Missed immunisation opportunities according to health facility

\begin{tabular}{llllllllllll}
\hline $\begin{array}{l}\text { Health } \\
\text { facility }\end{array}$ & BCG & OPVO & OPVI & OPV2 & OPV3 & DPTI & DPT2 & DPT3 & Measles & Total \\
\hline AMREF & $4(5.1)$ & 0 & 0 & 0 & 0 & 0 & 0 & 0 & 0 & 4 \\
Baba Dogo & $1(1.8)$ & 0 & $4(7.4)$ & $5(9.6)$ & $9(18.8)$ & 0 & $2(3.8)$ & $1(2.1)$ & $1(4)$ & 23 \\
Kayole & $1(1.2)$ & 1 & $11(18.0)$ & $11(23.9)$ & $4(10.3)$ & $1(1.6)$ & 0 & 0 & 0 & 29 \\
Mbagathi & $2(3.1)$ & 0 & 0 & 0 & 0 & $1(1.7)$ & 0 & 0 & $1(12.5)$ & 4 \\
Mukuru & $8(11.3)$ & 1 & 0 & 0 & 0 & 0 & 0 & 0 & 0 & 10 \\
Pangani & $4(6.1)$ & 1 & 0 & 0 & 0 & 0 & 0 & 0 & $2(14.3)$ & 7 \\
\hline Total & $20(4.8)$ & 3 & $15(4.4)$ & $16(5.5)$ & $14(5.7)$ & $2(0.6)$ & $2(0.7)$ & $1(0.4)$ & $4(4.1)$ & 77 \\
\hline
\end{tabular}

Parantheses show missed opportunities for immunisation as proportion of children eligible for each antigen who attended the health facilities.

Missed immunisation opportunities for the DPT series were fewer than for the OPV series. Missed opportunities for DPT1 occurred in Kayole and Mbagathi only where the proportions were similar at $1.6 \%$ and $1.7 \%$ respectively. Only Kayole had missed to immunise children who were eligible for DPT2 and DPT3, and the proportion of missed immunisation opportunities in Baba Dogo were 4\% and 2\% respectively. There were two children who were missed for measles immunisation in Pangani, representing $14 \%$ of eligible children who had presented at the facility for measles vaccination. The missed measles immunisation opportunities for Mbagathi and Baba Dogo were $13 \%$ and $4 \%$ respectively.

Missed immunisation opportunities were assessed according to reason for visit to the health facility (Table 3). Eighty four percent of missed opportunities occurred while children were brought to clinics for vaccination. Only $4 \%$ of missed opportunities occurred while child came to the clinic for curative services.

Table 3

Missed immunisation opportunities by reason of visit

\begin{tabular}{llllllllllll}
\hline $\begin{array}{l}\text { Reason for } \\
\text { visit }\end{array}$ & BCG & OPV0 & OPV1 & OPV2 & OPV3 & DPT1 & DPT2 & DPT1 & Measles & Total & $\%$ \\
\hline Vaccination & 19 & 1 & 15 & 15 & 8 & 2 & 2 & 0 & 3 & 65 & 84.4 \\
$\begin{array}{l}\text { Treatment } \\
\text { Other }\end{array}$ & 1 & 0 & 0 & 0 & 1 & 0 & 0 & 0 & 1 & 3 & 4.0 \\
\hline Total & 0 & 2 & 0 & 1 & 5 & 0 & 0 & 1 & 0 & 9 & 11.6 \\
\hline
\end{tabular}

Other - Includes weighing, accompanying other child and normal check up.

Mothers were asked the reasons why their children were not vaccinated. Only 20 mothers enumerated reasons they claimed health workers gave for not vaccinating children who came for immunisation (Table 4).

Table 4

Reasons given for non-vaccination

\begin{tabular}{lll}
\hline Reason for failure to immunise & No. of Observations & Proportion (\%) \\
\hline & & 20 \\
Vaccine was out of stock & 4 & 35 \\
Vaccine scheduled not to be given that day & 7 & 20 \\
Child was sick or under-weight & 4 & 15 \\
Child not yet of age & 3 & 10 \\
BCG syringe out of stock & 2 & 100 \\
\hline Total & 20 & 20 \\
\hline
\end{tabular}


Of the 20 responses mothers gave, scheduling of immunisation to other days was the most common reason $(35 \%)$. The refusal to immunise ill or underweight children was the reason given by four mothers, including not immunising because a child had a rash. Four mothers claimed children were not immunised because BCG vaccine and OPV were out of stock.

\section{DISCUSSION}

In the facilities covered by this study, immunisation coverage was higher for the DPT series (all above 85\%) compared to the OPV series ( $83 \%$ and below). There were more incorrectly administered OPVI and OPV2 vaccine doses, compared to corresponding doses of DPT. This partly explains the relatively lower coverage for the OPV series compared to the DPT series.

The lower immunisation coverage for OPV doses, had also to do with missed immunisation opportunities arising from a shortage of OPV that was reported in the period preceding to and during the survey. Interestingly, the $80 \%$ immunisation coverage for measles was higher than that for OPV3.

The overall missed immunisation opportunities rate in this study was $3 \%$. Compared to one recent and several old studies from Africa, this was similar to rates found in preventive settings in Zimbabwe (4\%) and Comoros $(5 \%)(9,10)$. However, it was lower than findings in Congo (13\%), South Africa (11\% and 16\%), Ethiopia (41\%), and Egypt (30\%) (11-15). Eighty four percent of the missed immunisation opportunities occurred when a child was brought for vaccination. Most missed opportunities for OPV immunisation occurred in Baba Dogo and Kayole health centres. This was mainly because these facilities had a shortage of OPV stocks, which was more pronounced in Kayole during the survey period. Of the 33 children who came for treatment as a primary reason for visiting the facilities, only three of those eligible for vaccination were not immunised at those health facilities. The $4 \%$ missed opportunities rate for measles in this study was lower than estimates recorded elsewhere which ranged from $9.8 \%$ to $80 \%$ (16-20).

Avoidance of inappropriately administered doses of vaccine and missed immunisation opportunities would have increased immunisation coverage for all antigens, with an increase of measles immunisation coverage by $19 \%$. Inappropriately administered immunisation and missed opportunities points to a gap in health worker training and systems of supply of vaccines that requires health policy to address. This would call for a reappraisal of routine supervision and training within relevant city facilities so that the primary schedule is understood and practiced by health providers.

Inappropriately timed or early vaccination is a main issue that came out of this study. We used the cut-off recommended by KEPI to determine ages of vaccination and intervals between doses, so that the age cut-off for, for example, OPV1/DPT1 administration was six weeks (42 days), and the interval between doses in a series was four weeks (28 days). What arises then is whether routine KEPI supervision or practitioners stress the exactness of immunisation timing. It is conceivable that EPI service providers at the facility level take the age cut-off in approximate rather than absolute terms so that 42 days would be "about 42 ". If the latter is practised it opens the way for different provider interpretations and a variation in practice by creating a "best judgement" scenario, which is not ideal from a programmatic point of view. The dilemma with absoluteness of age cut-offs to the health professional would, of course be choosing between turning back a child who is say 37 days old, without giving OPV1/ DPT1 and risking the mothers never bringing back that child for vaccination on the appropriate date.

A strengthened routine supervision during which EPI personnel are updated on the need to follow the recommended schedule may result in fewer inappropriate doses. As the policy of vaccinating children at all contacts is in place in all facilities country wide, the issue on the existence of missed immunisation opportunities points to some instances in practice where EPI workers do not check for children who are eligible for vaccination. The change here would be for managers or matrons of facilities to ensure that all contacts with children eligible for vaccination are utilised to increase vaccine coverage. Social mobilisation through community based organisations (CBOs) and other groups involved in health within the slums should be strengthened in order to impart more knowledge to mothers regarding the importance of routine vaccination.

\section{ACKNOWLEDGEMENTS}

To Prof. Felicity Cutts and Phillippa Cumberland for their contribution towards this study. This work was supported by a grant from the World Health Organisation, APW reference number 18-181-751.

\section{REFERENCES}

1. Expanded Programme on Immunisation. Global Advisory Group. I. Global Programme. Weekly Epidem. Record. 1986; 61:13-14

2. Galazka, A.M., Lauer, B.A., Henderson, R.H., and Keja, J. Indications and contraindications for vaccines used in the Expanded Programme on Immunisation. Bull. World Hlth. Org. 1984; 62:357-366.

3. Begg, N. and Nicoll, A. Immunisation. Brit. Med. J. 1994; 309:1073-1075.

4. Global Programme for Vaccines and Immunization, Expanded Programme on Immunization. Immunization Policy. WHO/EPI/GEN/95.03. Rev 1. 1996

5. Expanded Programme on Immunization. The Immunological Basis for Immunization. WHO documents WHO/EPI/GEN/ 93. 12-93. Module 1. General Immunology (A. Galazka); Module 5: Tuberculosis (J. Milstien); Module 6: Poliomyelitis 
(S. Robertson); Module 7: Measles (F. Cutts); Module 8. Yellow fever (S. Robertson). 1993.

6. Expanded Programme on Immunisation. Protocol for the assessment of missed opportunities for immunisation. WHO/ EPI/GEN/88. 6. 1988a.

7. Hutchins, S.S., Janse, H.A.F.M., Robertson S.E., Evans, P. and Kim-Farley R.J.. Studies of missed opportunities in developing and industrialised countries. Bull. World Hlth. Org. 1993; 71:549-560.

8. Expanded programme on Immunisation. Kenya. WHO/EPI/ GEN/83. 1983

9. McCmormic, C. Biellick, R., Shearley, A, and Saruchera H. Missed opportunities for vaccination in Chitungwiza, Zimbabwe 1995: a service deficiency which can easily be eliminated. South Afr. Med. J. 1996; 42:236-240.

10. Expanded programme on Immunisation. Missed immunisation opportunities. Comoros. Weekly Epidem. Record 1988b; 63:344-346.

11. Talani, P., Nkounkou,-P.J., Mayanda, H. and Yala, F. Missed vaccination opportunities. Soc. Exot. 2000; 93:121-122.

12. Bachmann, M.O. and Barron, P. Missed opportunities for immunisation in curative and preventive services in a community health centre. A follow-up survey. South Afr. Med. J. 1996; 86:947-949.
13. Harrison, D., Barron P., Glass B., Sonday, S. and Heyde Y. Far fewer missed opportunities in an integrated child health service. South Afr. Med. J. 1993; 83:575-576.

14. Expanded programme on Immunisation. Missed immunisation opportunities. Ethiopia. Weekly Epidem. Record. 1990; 65:167-170.

15. Expanded programme on Immunisation. Missed immunisation opportunities. Egypt. Weekly Epidem. Record. 1989; 64:93-94.

16. Cutts, F.T. Strategies to improve immunisation services in urban Africa. Bull. World Hlth Org. 1991; 69:407-414.

17. Gindler, J.S., Cutts, F.T, Antinori-Barnett, M.E., et al. Successes and failures in vaccine delivery: evaluation of the immunisation delivery system in Puerto Rico. Paediatrics 1993; 91:315-320.

18. Kahn, J.G., Mokdad, A.H., Deming, M.S., et al. Avoiding missed opportunities for immunisation in Central African Republic: potential impact on vaccination coverage. Bull. World Hlth Org. 1995; 73:47-55.

19. Money, M.K. and Mohan, P. Measles immunisation coverage in urban slums. Indian J. Paediat. 1999; 66:505-509.

20. Aswar, N.R. Deotale, P.G., Kale, K.M., Bhawalkar, J.S. and Dhage, V.R. Sociomedical correlates of missed opportunities for immunisation. Indian J. Public Health. 1999; 43:148-151. 